\title{
White Matter Fibertracking in First-Episode Schizophrenia, Schizoaffective Patients and Subjects at Ultra-High Risk of Psychosis
}

\author{
Bart D. Peters ${ }^{a} \quad$ Lieuwe de Haan $^{a} \quad$ Nienke Dekker ${ }^{a} \quad$ Jorik Blaas ${ }^{b}$ \\ Hiske E. Becker ${ }^{a}$ Peter M. Dingemans ${ }^{a}$ Erik M. Akkerman ${ }^{b}$ Charles B. Majoie $^{b}$ \\ Therèse van Amelsvoort $^{a}$ Gerard J. den Heeten ${ }^{b}$ Don H. Linszen ${ }^{a}$ \\ Departments of a Psychiatry and ${ }^{\mathrm{b}}$ Radiology, Academic Medical Center, Amsterdam, The Netherlands
}

\section{Key Words}

Schizophrenia, first-episode • Psychosis, ultra-high risk • Diffusion tensor imaging • Fibertracking • White matter

\begin{abstract}
There is increasing evidence of white matter pathology in schizophrenia. The aim of this study was to examine whether white matter abnormalities found with diffusion tensor imaging (DTI) in previous schizophrenia studies are present in the early phase of the illness. DTI was performed at 3 T on 10 male patients with a first $(n=8)$ or second $(n=2)$ psychotic episode of schizophrenia or schizoaffective disorder, 10 male patients at ultra-high risk of psychosis with (pre)psychotic symptoms and 10 healthy controls. Fibertracts found to be abnormal in other DTI studies (uncinate and arcuate fasciculus, anterior and dorsal cingulum, subdivisions of the corpus callosum) were calculated and visualized; tract-specific measurements (fractional anisotropy and trace) were performed. No differences were found between the healthy subjects and the 2 patient groups. These preliminary findings suggest that there is no white matter pathology of these association tracts detectable with DTI in the early stages of schizophrenic illness in males. Our findings are in contrast with DTI abnormalities found in some
\end{abstract}

other first-episode studies. This discrepancy in findings may be related to differences in subject characteristics and DTI methodology. Possible effects of age, gender, level of education and illicit substance use on DTI findings in schizophrenia are discussed.

Copyright $\odot 2008$ S. Karger AG, Basel

\section{Introduction}

Abnormal brain development in schizophrenia is thought to lead to 'dysconnectivity' between brain areas associated with positive and negative symptom dimensions and cognitive dysfunctioning [1-3]. The neuroanatomical substrate for this dysconnectivity may be dysplastic white matter tracts as a result of abnormal development in utero [2], demyelination during adolescence [1, 3] and adulthood [3], or an arrest in the normal process of myelination during brain maturation in adolescence and middle age [3].

With diffusion tensor imaging (DTI), brain white matter structure can be assessed in a more detailed manner in vivo than with conventional MRI [4]. In DTI, the magnetic resonance signal is made sensitive to the movement (or diffusion) of water molecules. A DTI index

\section{KARGER}

Fax +41613061234 E-Mail karger@karger.ch www.karger.com
(C) 2008 S. Karger AG, Basel

0302-282X/08/0581-0019\$24.50/0

Accessible online at:

www.karger.com/nps
Bart D. Peters

Department of Psychiatry, Academic Medical Center, University of Amsterdam Meibergdreef 5

NL-1105 AZ Amsterdam (The Netherlands)

Tel. +31 20891 3500, Fax +3120891 3701, E-Mail b.d.peters@amc.uva.nl 
called fractional anisotropy (FA) is increased by myelination $[5,6]$, coherence of fibertracts [7], and by the structural integrity of fibers, their diameter and packing density [4].

Subjects at ultra-high risk (UHR) of conversion to psychosis are characterized by defined trait or state factors, including attenuated or brief limited psychotic symptoms [8-10]. Studies in UHR subjects and first psychotic episode patients can provide insight into the early pathophysiological mechanisms in schizophrenia-like disorder.

Eight DTI studies in patients with a recent onset of schizophrenia have been reported so far [11-18], and these have reported abnormalities in several white matter areas. To our knowledge, no DTI studies in UHR patients have been reported. One diffusion-weighted study in first-degree relatives of schizophrenia patients found diffusion abnormalities in gray matter structures not detectable with conventional MRI [19]. In chronic schizophrenia patients, several DTI studies found reduced anisotropy in numerous brain regions [20-23]. One study found also increased anisotropy in a subgroup of patients [24].

Many DTI studies in schizophrenia examined regions of interest or used a voxel-by-voxel approach in grayscale anisotropy images, in which it can be difficult to locate abnormalities to specific fibertracts. Advanced applications of DTI make it possible to calculate and visualize the probable trajectories of white matter fiber bundles (referred to as 'fibertracking' [25]) and allow tract-specific measurements. To our knowledge, this is the first DTI study that applies fibertracking to examine white matter tracts in UHR patients as well as patients with a first or second psychotic episode of schizophrenia or a related disorder. Association fibertracts connecting cortical regions may be primarily involved in schizophrenia [26], and we hypothesized that FA of association fibertracts previously found to be abnormal in schizophrenia would be altered in both patient groups compared to healthy controls. Furthermore, antipsychotic medication use is a major confounder in schizophrenia research, and we hypothesized that there would be a positive correlation between the duration and dose of antipsychotic treatment and white matter FA, as found previously [22, 23]. Such a relation would be consistent with possible pro-myelination effects of some atypical antipsychotics, which have been found to increase brain neurosteroid and apolipoprotein D levels and blood lipid levels, which in turn are thought to stimulate myelination in the brain [3].

\section{Methods}

\section{Subjects}

This study was approved by the local and national medical ethics committees. Male patients diagnosed with a first or second psychotic episode of schizophrenia, schizoaffective disorder or schizophreniform disorder (referred to next as schizophrenia-like disorders) were recruited from the open-ward inpatient and day care units of the Adolescent Clinic of the Academic Medical Center. These patients were approached when they were deemed clinically able to give informed consent. UHR patients were recruited from a naturalistic longitudinal study program related to the Adolescent Clinic, with an 18month follow-up. These patients are referred by mental health services. Inclusion criteria for these patients were defined according to criteria used in other studies [8-10]: attenuated psychotic symptoms (e.g. odd beliefs, paranoid ideation) or brief psychotic moments with spontaneous remission in less than 1 week, and/or a decline in functioning in the past year (30-percent reduction in Global Assessment of Functioning scale) plus a genetic risk (first-degree relative with schizophrenia-like disorder or a schizotypal personality disorder), or 2 'basic symptoms' (cognitive, perceptual, emotional and social disturbances [10]). We included only males, as white matter abnormalities may be gender specific [27]. Male healthy control subjects were recruited through local advertisements, and were matched for educational level, age and handedness.

Exclusion criteria for all subjects were: history of a demonstrable neurological or endocrine disease that may affect the brain structure, history of a head trauma with loss of consciousness for more than $15 \mathrm{~min}$, mental retardation according to DSM-IV criteria (prior to onset of the first psychotic episode for schizophrenia-like patients), gross brain abnormalities on conventional MRI (other than atrophy or ventricular enlargement) and any standard exclusion criterion for MRI scanning (e.g. irremovable metal objects). Additional exclusion criterion for the UHR subjects was a current or past psychotic disorder. Additional exclusion criteria for healthy controls were: a personal or family history of a major psychiatric illness or a lifetime diagnosis of alcohol/other substance abuse or dependence. After a complete description of the study, written informed consent was obtained from all subjects.

\section{Clinical Measures}

Clinical discharge diagnoses (including substance abuse or dependence) of patients with schizophrenia-like disorder were made according to DSM-IV criteria by 2 clinical psychiatrists with the use of all available clinical information, including a history taken from a significant other (Longitudinal Expert Assessment of Diagnosis procedure [28]). An estimation of illness duration, defined as the time between the start of the first psychotic episode and MRI scanning, was based on an interview and all medical records.

For the UHR patients, fulfillment of the inclusion criteria and diagnoses according to DSM-IV criteria were determined by a clinical psychiatrist and a research psychologist with the Structured Interview for Prodromal Syndromes [9] and the Bonn Scale for the Assessment of Basic Symptoms [10]. Estimates of total duration of antipsychotic medication use in both patient groups were based on an interview and all medical records. Positive, negative and general symptoms in the schizophrenia-like and UHR 
patients were assessed by trained raters with the Positive and Negative Syndrome Scale (PANSS).

Healthy controls were screened in a clinical psychiatric interview by a psychiatry resident. Handedness was determined with the Annett Handedness Questionnaire [29]. Educational level was defined according to the educational levels of the Dutch high school system: 'skilled professional training' (requires average or below average IQ), and Bachelor's or Master's level.

\section{DTI Protocol and Postprocessing}

DTI and $\mathrm{T}_{1}$-weighted MRI images were acquired on a Philips Intera 3-T whole-body MRI scanner (Philips Intera, Philips Medical Systems, Best, The Netherlands). First a localizer scan was done. Whole brain DTI images were then acquired using singleshot spin-echo echo-planar imaging. Slice orientation was (para)transversal, tilted slightly to coronal according to the line touching the inferior of the genu and inferior of the splenium of the corpus callosum. DTI acquisition was along 16 non-colinear directions, and each direction was scanned twice and then averaged to improve signal-to-noise ratio. Resolution of the DTI dataset was $3 \times 3.5 \times 2.2 \mathrm{~mm}^{3}$, no gap. Other parameters were: field of view: $230-256 \mathrm{~mm}$ (depending on head size), echo-time: $94 \mathrm{~ms}$, repetition time (TR): 4,831-6,248 ms (depending on the number of slices, which varied with head size; TR did not vary within a scan; this variation in TR did not affect DTI results, since the $\mathrm{T}_{1}$-weighting factor, which is governed by $\mathrm{TR}$, is divided out in the postprocessing of the DTI image data), diffusion sensitivities: $\beta=0$ and $\beta=1,000 \mathrm{~s} / \mathrm{mm}^{2}$, image matrix: $256 \times 256$. Scan time was about 6 min per subject for the diffusion series, total scan time was less than $30 \mathrm{~min}$.

Subjects were positioned with their heads thoroughly fixated by inserting cushions between their ears and the head coil. No motion artefact correction was performed, because scans were acquired back-to-back and no displacement was visually detected. Since DTI measurements were acquired with single-shot echo-planar imaging sequences, motion artefacts like blurring or ghosting did not occur. After reconstruction, the diffusionweighted images were transferred to a workstation, where eigenvalue and eigenvector maps of the diffusion tensor were calculated. FA images were reconstructed, including FA images color-coded for main diffusion direction ( $\mathrm{x}, \mathrm{y}$ or $\mathrm{z}$ ). The acquired diffusion tensor images formed a basis to construct a full-brain white matter tractography for each individual separately. The algorithms presented by Basser et al. [30] formed the basis for the construction of the pathways that follow the white matter fiber bundles in the brain. We used the fourth-order integration of Basser et al. through the interpolated tensor field to generate the fiber paths [30]. We chose a whole-brain seeding approach, where many individual fiber tracts are generated throughout the entire brain. The individual starting points are seeded uniformly over the brain, and then, using these as start locations, each fiber bundle is traced in both directions until a stopping condition is met. We used 2 criteria for the stopping condition. First, the FA in each voxel had to be at least 0.2 . This value was suggested as the optimal FA value for fibertracking by Mori et al. [31] and Kunimatsu et al. [32]. Secondly, the bending angle of the tract is not allowed to exceed $45^{\circ}$. These stopping criteria also serve to diminish noise-induced errors [30]. This process resulted in a tractography for each subject containing around 30,000 fibers, which were composed of around 1.2 million points in total.

\section{DTI Quantification}

We examined association fibertracts that showed abnormalities in previous DTI studies in schizophrenia patients: the dorsal cingulum $[33,14]$, the anterior cingulum $[34,12,35]$, the uncinate fasciculus $[36,24]$ and the arcuate fasciculus $[36,24]$, the genu of the callosum [24] and the splenium of the callosum [24, 37, 38].

DTI data of each individual subject were analyzed with inhouse-developed software: Diffusion Tensor Imaging Interactive (DTII) [39]. The software allows interactive extraction of fiber bundles through the positioning of 3 boxes; only the fiber bundle(s) that passes through all 3 boxes are extracted and visualized. DTII allows visualization of the fibertracts overlaid on the anatomical $\mathrm{T}_{1}$-weighted MRI images and the (color-coded) FA images, in 1 or more slice views. In accordance with hypotheses that FA reduction in schizophrenia-like disorder results from myelination abnormalities [1,3], FA and the 'trace' (the average diffusion in all 3 main directions) were measured in the most 'homogeneous' part of each fiber bundle, i.e. the thickest part with the least branching off of small fibers. Fasciculi were segmented and visualized in the following manner. One box was placed in the 'starting point' of the fasciculus, 1 box was placed in the 'end point', and the measurement box was placed between the other 2 boxes. Mean FA and trace of the section of the fibertract in the measurement box were measured. Specifics on the boundaries used for each bundle are described below. To locate the bundles, the following resources were used: publications of studies on these bundles in schizophrenia patients, two DTI atlases $[25,40]$, one neuropathological atlas [41]. For reliability purposes, the size of the 3 boxes was maintained the same across subjects for each bundle. The spatial position of the boxes was defined by their location relative to structures in the $T_{1}$-weighted scan, to the bundle itself and to the other boxes.

Before actual measurements, inter-rater reliability for each bundle was determined in 10 subjects. The intra-class correlation (ICC) was calculated, and was required to be 0.90 or more for each fibertract before actual measurements were performed. Actual measurements were then done by 1 rater (B.D.P.). Boundaries of the measured bundles and their ICC were as follows:

Corpus Callosum. Figure 1 shows the corpus callosum, and how, in a similar way to Brambilla et al. [42], we divided the corpus callosum into 4 regions: splenium (posterior one third of the midsagittal length), genu (anterior one third), and anterior truncus and posterior truncus (each a half of the middle third). First, the measurement box (5.6-mm wide, left-right direction) was placed in the middle of the corpus callosum, where its fibers cross from the left to right hemisphere. The 2 other boxes were then placed adjacent to the measurement box, each one covering the whole left or right hemisphere; this visualized the complete corpus callosum. The sagittal view of the $\mathrm{T}_{1}$ scan was then placed in the middle of the corpus callosum. Next, the measurement box was resized to the length of the corpus callosum in the $\mathrm{T}_{1}$ scan. The anterior-posterior lengths of the measurement box were, for the splenium, posterior truncus, anterior truncus and genu, 2.6, $1.3,1.3$ and $2.6 \mathrm{~cm}$, respectively. The height (superior-inferior) was minimized to exclude the fornix from the measurements. Inter-rater reliability (ICC) was 0.95 for the total corpus callosum, 0.97 for the splenium, 0.97 for the posterior truncus, 0.93 for the anterior truncus and 0.94 for the genu.

Uncinate Fasciculus. Figure 2 shows the uncinate fasciculus, which connects the orbitofrontal lobe to the temporal pole. The 


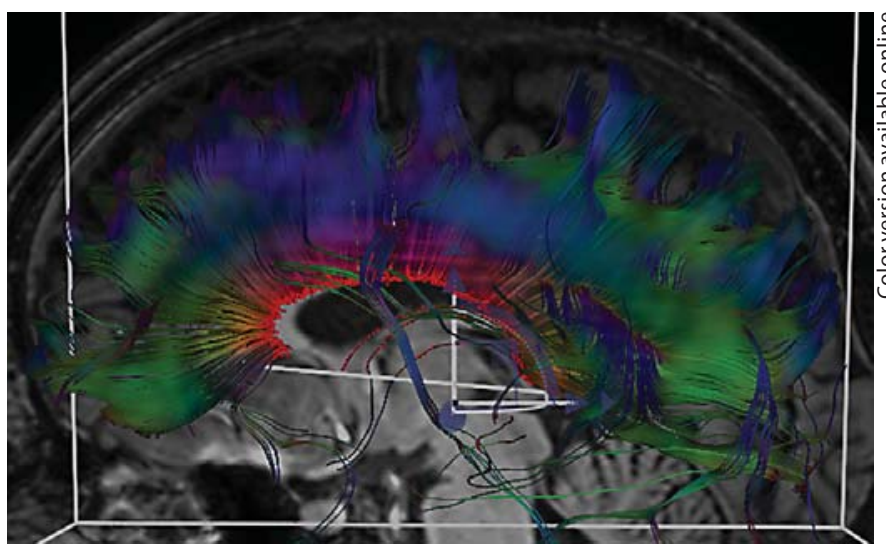

Fig. 1. The corpus callosum in a UHR subject. FA and trace were measured in the midsagittal part.

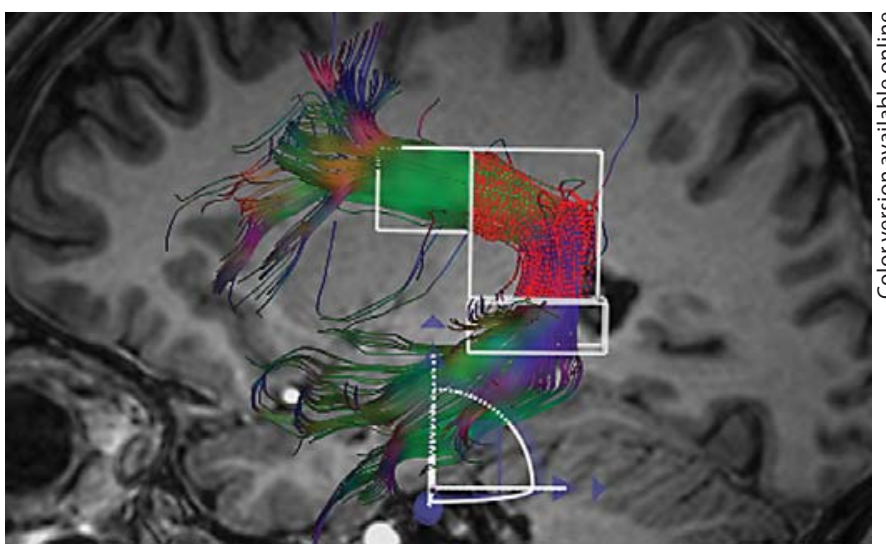

Fig. 3. The arcuate fasciculus in a UHR subject. FA and trace were measured in the middle box.

midsagittal slice of the $T_{1}$ structural MRI was used for reference. The uncinate was traced and measured by placing one box in the orbitofrontal lobe, another box in the temporal pole, and the measurement box $\left(3.9 \mathrm{~cm}^{3}: 1.4 \times 1.5 \times 1.9 \mathrm{~cm}\right)$ in the segment of the fasciculus containing the vertical (inferior to superior) fibers in the temporal pole and the first part of the horizontal fibers in the frontal lobe. ICC was 0.96 for the left and 0.92 for the right.

Arcuate Fasciculus. Figure 3 shows the arcuate fasciculus, which connects each frontal lobe with its hemisphere. One box was placed in the frontal part and the other box in the temporal part, both clearly identified in the coronal plane of the color-coded FA images. The measurement box $\left(10.2 \mathrm{~cm}^{3}: 2.3 \times 1.7 \times 2.7\right.$ $\mathrm{cm}$ ) was placed in the parietotemporal part of the long fibers, where the horizontal parietal part of the bundle curves downward vertically to the temporal lobe. ICC for the left was 0.97 and for the right 0.98 .

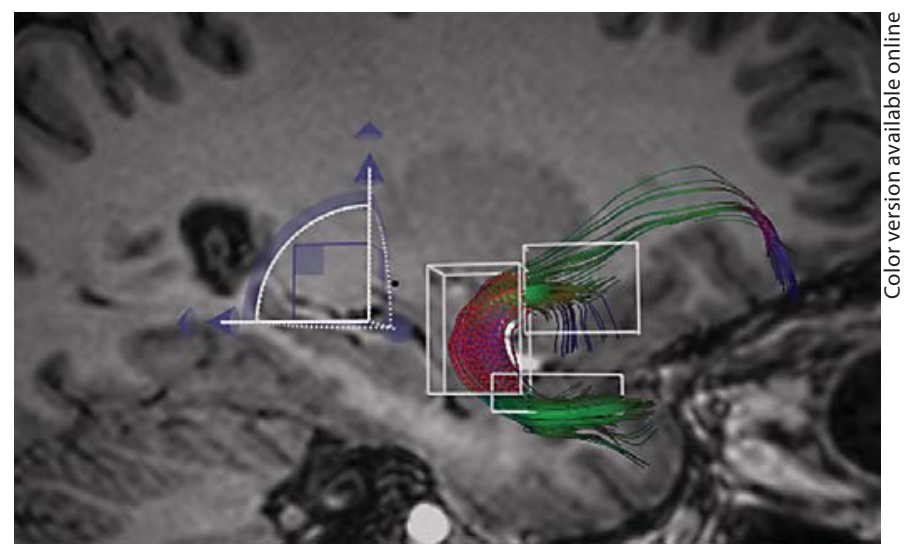

Fig. 2. The uncinate fasciculus in a UHR subject. FA and trace were measured in the middle box.

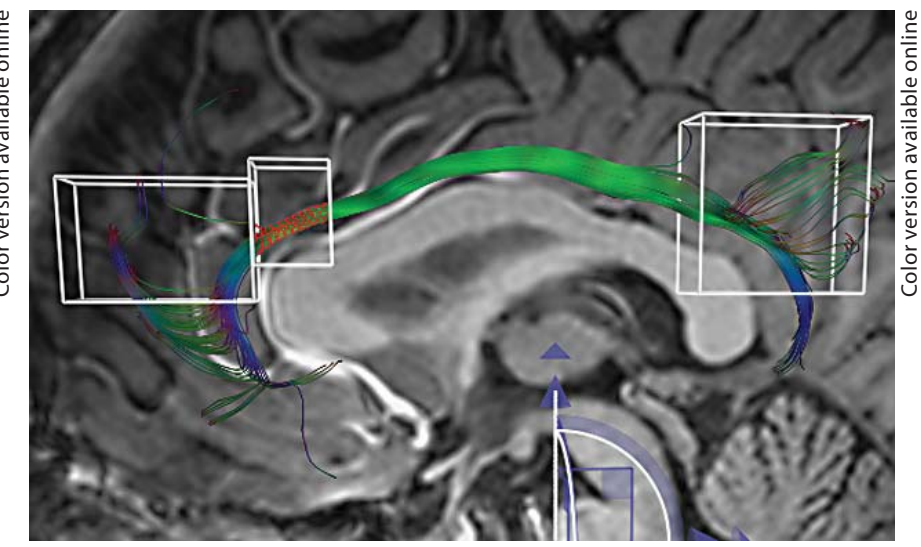

Fig. 4. The anterior cingulum in a healthy comparison subject. FA and trace were measured in the middle box.

Dorsal Cingulum. The cingulum runs from the frontal lobe to the temporal lobe and interconnects parts of the limbic system. The midsagittal slice of the $\mathrm{T}_{1}$ structural MRI was used for reference. One box was placed just anterior to the genu of the corpus callosum, another on the splenium of the corpus callosum. The measurement box was $40 \mathrm{~mm}$ long from anterior to posterior $(9.0$ $\mathrm{cm}^{3}: 4.0 \times 1.6 \times 1.4 \mathrm{~cm}$ ), and was placed with its anterior side to the dorsal side of the genu of the corpus callosum, which is in accordance with Kubicki et al. [33]. It was then moved superiorly to include the dorsal cingulum. ICC was 0.99 for the left and 0.94 for the right.

Anterior Cingulum. Figure 4 shows the anterior cingulum. The midsagittal slice of the $\mathrm{T}_{1}$ structural MRI was used for reference. The measurement box $\left(2.3 \mathrm{~cm}^{3}: 1.1 \times 1.6 \times 1.3 \mathrm{~cm}\right)$ was placed with its posterior side to the dorsal side of the genu of the corpus callosum. The other box was placed just anterior to the measurement box and the third box was placed on the splenium 
Table 1. Characteristics of patients and healthy controls

\begin{tabular}{lllc}
\hline & $\begin{array}{l}\text { First-episode } \\
\text { patients } \\
(\mathrm{n}=10)\end{array}$ & $\begin{array}{l}\text { UHR } \\
\text { patients } \\
(\mathrm{n}=10)\end{array}$ & $\begin{array}{l}\text { Healthy } \\
\text { controls } \\
(\mathrm{n}=10)\end{array}$ \\
\hline Age, years & $21.2 \pm 3.0$ & $21.6 \pm 2.8$ & $21.1 \pm 2.8$ \\
Educational level & $3 / 4 / 3$ & $3 / 2 / 5$ & $2 / 4 / 4$ \\
Handedness R/L $_{\text {Age at onset, years }}$ & $8 / 2$ & $10 / 0$ & $8 / 2$ \\
Antipsychotic medication & $20.1 \pm 2.7$ & - & - \\
$\quad$ Duration, weeks & 10 & 3 & - \\
Dose $^{2}$, mg/day & $33.5(7.1-97)$ & $14(10,12$ and 20$)$ & - \\
\hline
\end{tabular}

Figures are numbers, means \pm SD or medians with ranges in parentheses.

${ }^{1}$ Professional skilled training/Bachelor's level/Master's level.

${ }^{2}$ At the time of MRI scanning. Units are $\mathrm{mg} /$ day in haloperidol equivalents; 2 patients using quetiapine were excluded (estimation of the haloperidol equivalent of quetiapine was not deemed valid). of the corpus callosum; this corresponds to the procedure of Wang et al. [34]. The measurement box was then moved superiorly to cover the anterior cingulum. ICC was 0.94 for the left and 0.90 for the right.

\section{Statistical Analyses}

Data were analyzed using SPSS version 11.5. Repeated-measures analysis of variance (ANOVA) with group as the betweensubjects factor was used to test for differences in FA and trace between groups. The uncinate fasciculus and arcuate fasciculus were analyzed with side and fibertract (uncinate and arcuate) as within-subjects factors. The cingulum was analyzed with side and part of the cingulum (dorsal or anterior) as within-subjects factors. The corpus callosum was analyzed with the 4 sub-regions as within-subjects factors. In the case of a significant group or hemisphere effect or a group-by-hemisphere interaction, independent $t$ tests were used to compare group differences, and paired t tests were used to test for hemispheric asymmetry. The level of significance was set at $\mathrm{p}<0.05$.

Associations between illness duration, antipsychotic medication dose and duration, PANSS scores and diffusion measurements were calculated with Spearman's rank correlation $\left(r_{s}\right)$. A Bonferroni correction was applied for number of comparisons (i.e. fibertract measurements), setting the level of significance for correlations at $\mathrm{p}<0.05 / 12(\mathrm{p}=0.004)$.

\section{Results}

Ten patients with schizophrenia-like disorder, $10 \mathrm{UHR}$ patients and 10 controls were included (table 1). Eight schizophrenia-like patients were diagnosed with schizophrenia, and 2 with schizoaffective disorder. Eight patients experienced a first psychotic episode and 2 patients a second (all are referred to as first-episode patients in the following text). There were 3 patients with a history of cannabis dependence, 2 with cannabis abuse, 1 with cannabis dependence and amphetamine abuse, 1 with cannabis dependence and cocaine abuse and alcohol abuse. Eight patients used atypical antipsychotic medication and 2 used typical antipsychotic medication. Median duration of illness was 0.9 years (range: $0.3-2.6$ ).

The DSM-IV diagnoses of the UHR patients were: depressive disorder $(n=2)$, schizoid personality disorder $(\mathrm{n}=1)$ and preliminary diagnosis of bipolar disorder $(\mathrm{n}=$ 1); 6 received no diagnosis. Nine UHR patients experienced attenuated positive symptoms, of whom 3 also experienced brief limited intermittent psychotic symptoms and 2 had a first-degree relative with schizophrenia-like disorder plus reduced functioning; 1 patient had (pre)psychotic basic symptoms (thought blockages and unstable ideas of reference). Three UHR patients had used antipsychotic medication (1 risperidone and 2 olanzapine). One patient used an antihistamine drug, 1 had used antidepressants, 1 had used benzodiazepines, 1 had used methylphenidate as a child. Two patients had a history of cannabis abuse, and 1 a history of amphetamine, XTC (3,4 methylene-dioxymethamphetamine) and cannabis abuse.

There were no significant differences between the groups in age or educational level.

There were no differences in FA or trace between the first-episode patients, UHR subjects and controls in any of the fibertracts (table 2).

There was a significant effect of hemisphere in FA $(\mathrm{F}=44.84$, d.f. $=1, \mathrm{p}<0.001)$ and trace $(\mathrm{F}=5.35$, d.f. $=$ $1, \mathrm{p}=0.03)$ of the cingulum in controls and patients grouped together. Follow-up paired t tests showed that in 
Table 2. White matter DTI measurements in 4 association fibertracts

\begin{tabular}{|c|c|c|c|c|c|c|}
\hline & $\begin{array}{l}\text { First-episode } \\
\text { patients } \\
(\mathrm{n}=10)\end{array}$ & $\begin{array}{l}\text { UHR } \\
\text { patients } \\
(\mathrm{n}=10)\end{array}$ & $\begin{array}{l}\text { Healthy controls } \\
(\mathrm{n}=10)\end{array}$ & $\mathrm{F}$ & d.f. & $\mathrm{p}$ \\
\hline \multicolumn{7}{|l|}{ Uncinatus } \\
\hline \multicolumn{4}{|l|}{ Fractional anisotropy } & \multirow[t]{3}{*}{0.15} & \multirow[t]{3}{*}{2} & \multirow[t]{3}{*}{0.86} \\
\hline Left & $0.479 \pm 0.050$ & $0.477 \pm 0.044$ & $0.477 \pm 0.053$ & & & \\
\hline Right & $0.467 \pm 0.034$ & $0.494 \pm 0.044$ & $0.485 \pm 0.041$ & & & \\
\hline \multicolumn{4}{|l|}{ Trace } & \multirow[t]{3}{*}{0.35} & \multirow[t]{3}{*}{2} & \multirow[t]{3}{*}{0.71} \\
\hline Left & $0.248 \pm 0.014$ & $0.249 \pm 0.008$ & $0.244 \pm 0.009$ & & & \\
\hline Right & $0.245 \pm 0.006$ & $0.239 \pm 0.007$ & $0.245 \pm 0.005$ & & & \\
\hline \multicolumn{7}{|l|}{ Arcuatus } \\
\hline \multicolumn{4}{|l|}{ Fractional anisotropy } & \multirow{3}{*}{\multicolumn{3}{|c|}{ (analyzed with uncinatus) }} \\
\hline Left & $0.503 \pm 0.031$ & $0.501 \pm 0.029$ & $0.508 \pm 0.035$ & & & \\
\hline Right & $0.502 \pm 0.046$ & $0.505 \pm 0.025$ & $0.503 \pm 0.027$ & & & \\
\hline Trace & & & & \multirow{3}{*}{\multicolumn{3}{|c|}{ (analyzed with uncinatus) }} \\
\hline Left & $0.226 \pm 0.013$ & $0.225 \pm 0.008$ & $0.228 \pm 0.006$ & & & \\
\hline Right & $0.224 \pm 0.011$ & $0.222 \pm 0.009$ & $0.266 \pm 0.007$ & & & \\
\hline \multicolumn{7}{|l|}{ Cingulum } \\
\hline \multicolumn{4}{|l|}{ Fractional anisotropy } & \multirow[t]{5}{*}{1.21} & \multirow[t]{5}{*}{2} & \multirow[t]{5}{*}{0.31} \\
\hline Dorsal left & $0.536 \pm 0.072$ & $0.572 \pm 0.053$ & $0.561 \pm 0.043$ & & & \\
\hline Dorsal right & $0.498 \pm 0.049$ & $0.563 \pm 0.049$ & $0.516 \pm 0.043$ & & & \\
\hline Anterior left & $0.483 \pm 0.060$ & $0.498 \pm 0.055$ & $0.496 \pm 0.047$ & & & \\
\hline Anterior right & $0.437 \pm 0.063$ & $0.480 \pm 0.060$ & $0.409 \pm 0.049$ & & & \\
\hline \multicolumn{4}{|l|}{ Trace } & \multirow[t]{5}{*}{0.92} & \multirow[t]{5}{*}{2} & \multirow[t]{5}{*}{0.41} \\
\hline Dorsal left & $0.250 \pm 0.028$ & $0.245 \pm 0.012$ & $0.243 \pm 0.010$ & & & \\
\hline Dorsal right & $0.243 \pm 0.016$ & $0.232 \pm 0.009$ & $0.242 \pm 0.007$ & & & \\
\hline Anterior left & $0.254 \pm 0.032$ & $0.247 \pm 0.009$ & $0.240 \pm 0.011$ & & & \\
\hline Anterior right & $0.248 \pm 0.024$ & $0.238 \pm 0.014$ & $0.245 \pm 0.011$ & & & \\
\hline \multicolumn{7}{|l|}{ Corpus Callosum } \\
\hline \multicolumn{4}{|l|}{ Fractional anisotropy } & \multirow[t]{5}{*}{1.67} & \multirow[t]{5}{*}{2} & \multirow[t]{5}{*}{0.21} \\
\hline Splenium & $0.680 \pm 0.028$ & $0.706 \pm 0.018$ & $0.680 \pm 0.020$ & & & \\
\hline Posterior truncus & $0.551 \pm 0.029$ & $0.553 \pm 0.045$ & $0.542 \pm 0.029$ & & & \\
\hline Anterior truncus & $0.558 \pm 0.040$ & $0.568 \pm 0.033$ & $0.564 \pm 0.027$ & & & \\
\hline Genu & $0.624 \pm 0.038$ & $0.651 \pm 0.039$ & $0.638 \pm 0.029$ & & & \\
\hline Trace & & & & 2.66 & 2 & 0.09 \\
\hline Splenium & $0.287 \pm 0.017$ & $0.285 \pm 0.014$ & $0.291 \pm 0.014$ & & & \\
\hline Posterior truncus & $0.320 \pm 0.011$ & $0.312 \pm 0.027$ & $0.332 \pm 0.014$ & & & \\
\hline Anterior truncus & $0.309 \pm 0.017$ & $0.295 \pm 0.020$ & $0.298 \pm 0.023$ & & & \\
\hline Genu & $0.284 \pm 0.019$ & $0.280 \pm 0.017$ & $0.292 \pm 0.025$ & & & \\
\hline
\end{tabular}

controls and patients grouped together there was a left $>$ right asymmetry of FA in the dorsal $(t=5.75$, d.f. $=$ $29, \mathrm{p}<0.001)$ and anterior $(\mathrm{t}=4.45$, d.f. $=29, \mathrm{p}<0.001)$ cingulum, and a left $>$ right asymmetry of trace in the dorsal cingulum $(t=2.70$, d.f. $=29, \mathrm{p}=0.01)$.

As substance use by some patients was a possible confounder, post hoc analyses were performed to explore differences between patients with and without substance use. We applied a Bonferroni correction ( $\mathrm{p}=$ $0.05 / 12=0.004)$ as 12 new repeated measures ANOVA were performed [(cingulum, arcuate-uncinate, corpus callosum $) \times($ trace or FA $) \times($ UHR or first-episode patients)].

There were no differences in FA or trace between the first-episode patients with and without substance use. Similarly, UHR patients with substance use were compared with UHR patients without substance use. There were three UHR patients with a history of substance abuse. In addition, 1 patient was not diagnosed as such, but had used cannabis frequently for some time. There- 
fore, we compared the four UHR patients with substance (ab)use with the 6 patients without substance (ab)use. There was a trend for the corpus callosum $(\mathrm{F}=8.1$, d.f. $=$ $1, p=0.022$ ), where trace of the genu was higher in UHR patients with substance $(\mathrm{ab}) \mathrm{use}(\mathrm{t}=-2.9$, d.f. $=5.5, \mathrm{p}=$ 0.032; equal variances not assumed).

Further post hoc ANOVA comparing first-episode patients and UHR patients without substance use with controls showed that there was a trend for a difference in trace in the corpus callosum $(\mathrm{F}=6.2$, d.f. $=2, \mathrm{p}=0.010)$. In first-episode patients without substance use trace was lower in the splenium compared with controls $(t=4.4$, d.f. $=11.0, \mathrm{p}=0.002$; equal variances not assumed); in UHR patients without substance use, trace was lower in the posterior truncus $(\mathrm{t}=3.1$, d.f. $=7.6, \mathrm{p}=0.016$; equal variances not assumed) and the genu $(t=2.4$, d.f. $=13.5$, $\mathrm{p}=0.033$; equal variances not assumed) compared with controls. There were no differences in trace in the other fibertracts, nor FA differences, between the 2 patient groups without substance use and controls. Neither were there differences in trace or FA between the 2 patients groups with substance use and controls.

In first-episode patients, FA in the right arcuate fasciculus was nonsignificantly related to the duration of antipsychotic medication use $\left(\mathrm{r}_{\mathrm{s}}=-0.65, \mathrm{p}=0.043\right)$. When the three UHR patients with a history of antipsychotic use were included, the correlation became weaker $\left(\mathrm{r}_{\mathrm{s}}=\right.$ $-0.47, \mathrm{p}=0.103)$. There appeared a trend for a relation between FA in the right anterior cingulum and the duration of antipsychotic medication use $\left(\mathrm{r}_{\mathrm{s}}=-0.59, \mathrm{p}=\right.$ 0.033). There was no significant relation between FA or trace and dose of antipsychotic medication or duration of illness in any of the fibertracts.

In the patient groups combined, there was a trend for a correlation between positive symptom scores and FA of the left anterior cingulum $\left(\mathrm{r}_{\mathrm{s}}=0.53, \mathrm{p}=0.017\right)$. Trace in the left uncinate correlated with positive symptom scores $\left(\mathrm{r}_{\mathrm{s}}=0.51, \mathrm{p}=0.021\right)$, trace in the right uncinate with general and total symptom scores $\left(\mathrm{r}_{\mathrm{s}}=0.48, \mathrm{p}=0.030\right.$, and $\mathrm{r}_{\mathrm{s}}=0.47, \mathrm{p}=0.037$, respectively), and trace in the splenium of the corpus callosum with positive symptom scores $\left(\mathrm{r}_{\mathrm{s}}=0.47, \mathrm{p}=0.038\right)$.

\section{Discussion}

With diffusion tensor imaging fibertracking, we found no significant differences in FA or trace of 4 association tracts between first-episode patients with schizophrenia or schizoaffective disorder, UHR patients and control subjects. We did not find significant relations between FA or trace and antipsychotic medication use.

Our finding of a lack of DTI abnormalities in firstepisode patients contrasts with findings of reduced FA in other first-episode or recent-onset studies. Hao et al. [12] found reduced FA in the anterior cingulum, Karlsgodt et al. [18] found reduced FA in the arcuate fasciculus, and Szeszko et al. [17] found abnormalities approximately corresponding to the arcuate fasciculus and uncinate fasciculus. However, Price et al. [15] applied fibertracking and found no differences between recent-onset patients and controls in mean FA in the uncinate fasciculus. The spread of the FA distribution along the tract was skewed in the patients, which is thought to represent reduced FA in the core of the tract. In accordance with another first-episode study [13], we found no abnormalities in the genu or splenium of the corpus callosum. In contrast, Cheung et al. [16] did find reduced FA in the splenium of the corpus callosum in medication-naive firstepisode patients.

There may be several explanations for this discrepancy in findings between DTI studies in young-adult patients with schizophrenia-like disorder. First, the lack of differences between patients and controls in our study may result from lack of power due to the small sample size. However, one first-episode study finding differences included 10 patients [11], whereas the study finding no differences included 20 patients [13].

Secondly, level of education of the subjects may be an important confounder, although subjects were matched carefully on educational level. In our study, 7 out of 10 first-episode patients and 7 out of 10 UHR patients had received education at a Bachelor or Master's level. A structural MRI study in first-episode patients with a high level of education found no differences compared with healthy controls in cerebral white matter volume [43], while other first-episode studies did [44, 45]. High educational level has been associated with good outcome [46, 47], which in turn has been associated with a relative lack of brain abnormalities at illness presentation [48] and in the course of the illness [49]. Educational level was not mentioned in 4 of the first-episode or recent-onset DTI studies [11, 13-15], and was somewhat higher in controls in 1 study [12]. Parental socioeconomic status or years of education were matched in 3 studies [16-18].

Third, a gender effect may be involved. We included males only, and the 7 DTI studies showing abnormalities included both males and females. Postmortem studies suggest that female patients have more white matter abnormalities than male patients $[50,51]$. It is well known 
that there are gender differences in brain anatomy, and this should be taken into account in MRI studies. However, the other negative study [13] also included males and females.

Fourth, there had been considerable use of illicit drugs in our group of first-episode patients, mainly cannabis. In the other studies, only sporadic cannabis use by patients was reported [14], or patients with substance dependence $[12,15]$ or abuse [11] or 'psychostimulant use' [16] were excluded; in 1 study, one third of patients had a comorbid diagnosis of substance abuse [17]; 2 studies did not report on illicit drug use by patients $[13,18]$. In another DTI study in a larger sample of first-episode patients, we found increased FA in the uncinate fasciculus, which was restricted to patients with a history of illicit drug use, in particular cannabis use before age 17 (Peters et al., in submission). Delisi et al. [52] found increased FA in young adult subjects with adolescent cannabis use. These findings could mean that adolescent cannabis use increases white matter anisotropy through some effect on brain development. Alternatively, schizophrenia patients with early cannabis use may represent a subgroup of patients with a distinct pathophysiology. Post hoc analyses in the present study showed virtually no differences between patients with and without substance use (mainly cannabis). It is therefore unlikely that our results were confounded by substance use. There was a trend that patients without substance use had lower trace in parts of the corpus callosum. DIT differences between schizophrenia patients with and without substance use are probably subtle, and should be studied further in larger samples.

Fifth, our recent-onset patients were younger (2.2-7.3 years on average) than the patients in all but one of the other first-episode DTI studies. Age was similar in 1 study [18]. Duration of illness of patients was shorter in 3 of these studies [14, 16, 17], similar to ours in another [12], slightly longer in another [18] and not mentioned in 2 studies. Perhaps increasing age during young adulthood in schizophrenia is accompanied by an abnormal decrease in FA due to abnormal brain maturation. This may be independent of any progressive brain abnormalities developing after onset of the first psychotic episode.

Finally, methodological differences in postprocessing and quantification of the DTI images may play a role in the conflicting results. Five first-episode studies [11, 12, $14,16,17]$ employed a voxel-by-voxel analysis. The accuracy of voxel-based techniques is limited by possible misclassification of brain tissue, errors in inter-subject co- registration [53], and possible type I errors of a large number of comparisons (although this was addressed in these studies with a cluster-size threshold). Karlsgodt et al. [18] performed region-of-interest measurements after employing an advanced tract-based intersubject co-registration method. Interestingly, the 2 first-episode studies employing fibertracking or a conventional region-ofinterest method found no differences in mean FA [13, $15]$.

In studies using conventional FA images, instead of fibertracking algorithms, measurements may be influenced by fibertracts crossing the tract of interest. For instance, measurements in the uncinate fasciculus are likely to include part of the anterior commissure and the inferior occipitofrontal fasciculus [41]. With our interactive fibertract selection with 3 boxes, we could easily segment the uncinate fasciculus from the inferior occipitofrontal fasciculus.

There were some trends for correlations between DTI indices and positive and general symptom scores, but not negative symptom scores. Whether white matter diffusion abnormalities are specifically related to positive symptoms and not negative symptoms should be further examined in larger samples.

Our fibertracking study has some limitations. Fibertracking is a relatively new tool to investigate white matter anatomy, and it is influenced in certain regions by crossing fibers. In addition, the voxels in our study were not collected isotropically, and isotropically acquired voxels are preferred for fibertracking. Also, the anatomical boundaries for our measurements were defined for the most part by the fibertracts; thus, using the dependent variable to define its boundaries. Furthermore, we did not measure fibertract diameter, and it is possible that there were differences in fibertract diameter between the groups. As to the quality of the DTI and fibertracking, we noticed that in all cases we could reconstruct fibertracts consisting of densely packed parallel fiber lines. Erroneous fiber lines, wandering away in unlikely directions, which signal the influence of noise, were very infrequent, and they were not taken into account in the measurement.

Until now, little has been done about the validation of DTI. Larger studies and especially neuroanatomical and pathological comparisons with brain specimens are lacking in this stage of research. Still, in the future it could provide us with an important part of the diagnostic chain, where we could link fibertracking to functional MRI and neurophysiological data and performance tests. 


\section{Conclusion}

To our knowledge, this is the first DTI study comparing young-adult schizophrenia and schizoaffective patients as well as UHR patients with healthy controls using white matter fibertracking. We found no DTI abnormalities in 4 association fibertracts in either patient group. Larger longitudinal studies including neuroleptic naïve patients are needed to determine the effect of age, illness duration, gender, illicit drug use and medication on DTI measurements in schizophrenia. Longitudinal studies in UHR patients can determine which DTI abnormalities are associated with transition to psychosis. More advanced imaging and quantification methods, in particular high-field-strength imaging with higher resolution and tract-specific measurements through fibertracking, may give better insight into which white matter pathways are implicated in the pathophysiology of schizophrenia.

\section{Acknowledgments}

The authors thank Dr. Harry Uylings (Department of Anatomy and Neuroscience, VU University Medical Center, Amsterdam) for his kind and insightful advice on the boundaries and trajectory of the uncinate fasciculus.

This study was funded by grant 28-1241-2 from the Dutch Health Research and Development (ZONMw).

\section{References}

1 Hyde TM, Ziegler JC, Weinberger DR: Psychiatric disturbances in metachromatic leukodystrophy. Arch Neurol 1992;49:401406.

2 Bullmore ET, Frangou S, Murray RM: The dysplastic net hypothesis: an integration of developmental and dysconnectivity theories of schizophrenia. Schizophr Res 1997;28: 143-156.

-3 Bartzokis G: Schizophrenia: breakdown in the well-regulated lifelong process of brain development and maturation. Neuropsychopharmacology 2002;27:672-683.

$\checkmark 4$ Beaulieu C: The basis of anisotropic water diffusion in the nervous system - a technical review. NMR Biomed 2002;15:435-455

$\checkmark 5$ Gulani V, Webb AG, Duncan ID, Lauterbur PC: Apparent diffusion tensor measurements in myelin-deficient rat spinal cords. Magn Reson Med 2001;45:191-195.

-6 Sakuma H, Nomura Y, Takeda K, Tagami T, Nakagawa T, Tamagawa Y, Ishii Y, Tsukamoto T: Adult and neonatal human brain: diffusional anisotropy and myelination with diffusion-weighted MR imaging. Radiology 1991;180:229-233.

$\checkmark 7$ Ono J, Harada K, Takahashi M, Maeda M, Ikenaka K, Sakurai K, Sakai N, Kagawa T, Fritz-Zieroth B, Nagai T, Nihei A, Hashimoto S, Okada S: Differentiation between dysmyelination and demyelination using magnetic resonance diffusional anisotropy. Brain Res 1995;671:141-148.

8 Yung AR, Phillips LJ, Yuen HP, Francey SM, McFarlane CA, Hallgren M, McGorry PD: Psychosis prediction: 12-month follow up of a high-risk ('prodromal') group. Schizophr Res 2003;60:21-32.
9 Miller TJ, McGlashan TH, Rosen JL, Somjee L, Markovich PJ, Stein K, Woods SW: Prospective diagnosis of the initial prodrome for schizophrenia based on the Structured Interview for Prodromal Syndromes: preliminary evidence of interrater reliability and predictive validity. Am J Psychiatry 2002; 159:863-865.

10 Klosterkotter J, Hellmich M, Steinmeyer EM, Schultze-Lutter F: Diagnosing schizophrenia in the initial prodromal phase. Arch Gen Psychiatry 2001;58:158-164.

-11 Szeszko PR, Ardekani BA, Ashtari M, Kumra S, Robinson DG, Sevy S, Gunduz-Bruce H: White matter abnormalities in first-episode schizophrenia or schizoaffective disorder: a diffusion tensor imaging study. Am J Psychiatry 2005;162:602-605.

12 Hao Y, Liu Z, Jiang T, Gong G, Liu H, Tan L, Kuang F, Xu L, Yi Y, Zhang Z: White matter integrity of the whole brain is disrupted in first-episode schizophrenia. Neuroreport 2006;17:23-26.

13 Price G, Bagary MS, Cercignani M, Altmann DR, Ron MA: The corpus callosum in first episode schizophrenia: a diffusion tensor imaging study. J Neurol Neurosurg Psychiatry 2005;76:585-587.

14 Federspiel A, Begre S, Kiefer C, Schroth G, Strik WK, Dierks T: Alterations of white matter connectivity in first episode schizophrenia. Neurobiol Dis 2006;22:702-709.

15 Price G, Cercignani M, Parker GJ, Altmann DR, Barnes TR, Barker GJ, Joyce EM, Ron MA. White matter tracts in first-episode psychosis: a DTI tractography study of the uncinate fasciculus. Neuroimage 2008;39: 949-955.
16 Cheung V, Cheung C, McAlonan GM, Deng Y, Wong JG, Yip L, Tai KS, Khong PL, Sham P, Chua SE: A diffusion tensor imaging study of structural dysconnectivity in never-medicated, first-episode schizophrenia. Psychol Med 2008;38:877-885.

17 Szeszko PR, Robinson DG, Ashtari M, Vogel J, Betensky J, Sevy S, Ardekani BA, Lencz T, Malhotra AK, McCormack J, Miller R, Lim KO, Gunduz-Bruce H, Kane JM, Bilder RM: Clinical and neuropsychological correlates of white matter abnormalities in recent onset schizophrenia. Neuropsychopharmacology 2008;33:976-984.

18 Karlsgodt KH, van Erp TG, Poldrack RA, Bearden CE, Nuechterlein KH, Cannon TD: Diffusion tensor imaging of the superior longitudinal fasciculus and working memory in recent-onset schizophrenia. Biol Psychiatry 2008;63:512-518.

19 Delisi LE, Szulc KU, Bertisch H, Majcher M, Brown K, Bappal A, Branch CA, Ardekani BA: Early detection of schizophrenia by diffusion weighted imaging. Psychiatry Res 2006;148:61-66.

-20 Kanaan RA, Kim JS, Kaufmann WE, Pearlson GD, Barker GJ, McGuire PK: Diffusion tensor imaging in schizophrenia. Biol Psychiatry 2005;58:921-929.

21 Okugawa G, Nobuhara K, Minami T, Takase K, Sugimoto T, Saito Y, Yoshimura M, Kinoshita T: Neural disorganization in the superior cerebellar peduncle and cognitive abnormality in patients with schizophrenia: a diffusion tensor imaging study. Prog Neuropsychopharmacol Biol Psychiatry 2006; 30:1408-1412. 
22 Minami T, Nobuhara K, Okugawa G, Takase K, Yoshida T, Sawada S, Ha-Kawa S, Ikeda K, Kinoshita T: Diffusion tensor magnetic resonance imaging of disruption of regional white matter in schizophrenia. Neuropsychobiology 2003;47:141-145.

23 Okugawa G, Nobuhara K, Minami T, Tamagaki C, Takase K, Sugimoto T, Sawada S, Kinoshita T: Subtle disruption of the middle cerebellar peduncles in patients with schizophrenia. Neuropsychobiology 2004; 50:119-123.

-24 Hubl D, Koenig T, Strik W, Federspiel A, Kreis R, Boesch C, Maier SE, Schroth G, Lovblad K, Dierks T: Pathways that make voices: white matter changes in auditory hallucinations. Arch Gen Psychiatry 2004;61: 658-668.

25 Wakana S, Jiang H, Nagae-Poetscher LM, van Zijl PC, Mori S: Fiber tract-based atlas of human white matter anatomy. Radiology 2004;230:77-87.

26 Pearlson GD, Petty RG, Ross CA, Tien AY: Schizophrenia: a disease of heteromodal association cortex? Neuropsychopharmacology 1996;14:1-17.

-27 Chance SA, Highley JR, Esiri MM, Crow TJ: Fiber content of the fornix in schizophrenia: lack of evidence for a primary limbic encephalopathy. Am J Psychiatry 1999;156:17201724 .

28 Spitzer RL, Williams JBW: Classification of mental disorders; in Kaplan HJ, Sadock BJ (eds): Comprehensive Textbook of Psychiatry, ed 4. Baltimore, Williams and Wilkins, 1985, pp 591-612.

29 Annett M: A classification of hand preference by association analysis. Br J Psychol 1970;61:303-321.

- 30 Basser PJ, Pajevic S, Pierpaoli C, Duda J, Aldroubi A: In vivo fiber tractography using DT-MRI data. Magn Reson Med 2000;44: 625-632.

- 31 Mori S, Kaufmann WE, Davatzikos C, Stieltjes B, Amodei L, Fredericksen K, Pearlson GD, Melhem ER, Solaiyappan M, Raymond GV, Moser HW, van Zijl PC: Imaging cortical association tracts in the human brain using diffusion-tensor-based axonal tracking. Magn Reson Med 2002;47:215223.

- 32 Kunimatsu A, Aoki S, Masutani Y, Abe O, Hayashi N, Mori H, Masumoto T, Ohtomo K. The optimal trackability threshold of fractional anisotropy for diffusion tensor tractography of the corticospinal tract. Magn Reson Med Sci 2004;3:11-17.
33 Kubicki M, Westin CF, Nestor PG, Wible CG, Frumin M, Maier SE, Kikinis R, Jolesz FA, McCarley R, Shenton ME: Cingulate fasciculus integrity disruption in schizophrenia: a magnetic resonance diffusion tensor imaging study. Biol Psychiatry 2003;54: 1171-1180.

34 Wang F, Sun Z, Cui L, Du X, Wang X, Zhang $\mathrm{H}$, Cong Z, Hong N, Zhang D: Anterior cingulum abnormalities in male patients with schizophrenia determined through diffusion tensor imaging. Am J Psychiatry 2004; 161:573-575.

35 Rose SE, Chalk JB, Janke AL, Strudwick MW, Windus LC, Hannah DE, McGrath JJ, Pantelis C, Wood SJ, Mowry BJ: Evidence of altered prefrontal-thalamic circuitry in schizophrenia: an optimized diffusion MRI study. Neuroimage 2006;32:16-22.

36 Burns J, Job D, Bastin ME, Whalley H, Macgillivray T, Johnstone EC, Lawrie SM: Structural disconnectivity in schizophrenia: a diffusion tensor magnetic resonance imaging study. Br J Psychiatry 2003;182:439-443.

- 37 Foong J, Maier M, Clark CA, Barker GJ, Miller DH, Ron MA: Neuropathological abnormalities of the corpus callosum in schizophrenia: a diffusion tensor imaging study. J Neurol Neurosurg Psychiatry 20006;8:242244.

-38 Agartz I, Andersson LR, Skare S: Abnormal brain white matter in schizophrenia: a diffusion tensor imaging study. Neuroreport 2001;12:2251-2254.

39 Blaas J, Botha CP, Peters BD, Vos F, Post FH: Fast and reproducible fiber bundle selection in DTI visualization. 16th IEEE Visualization, 2005, pp 59-64.

40 Catani M, Howard RJ, Pajevic S, Jones DK: Virtual in vivo interactive dissection of white matter fasciculi in the human brain. Neuroimage 2002;17:77-94.

41 Williams TH, Gluhbegovic N, Jew JY: The cerebral hemispheres; in Gluhbegovic N, Williams TH (eds): The Human Brain: A Photographic Guide. Hagerstown, Harper \& Row, 1980, chapter 5 .

42 Brambilla P, Cerini R, Gasparini A, Versace A, Andreone N, Vittorini E, Barbui C, Pelizza L, Nose M, Barlocco L, Marrella G, Gregis M, Tournikioti K, David AS, Keshavan MS Tansella M: Investigation of corpus callosum in schizophrenia with diffusion imaging. Schizophr Res 2005;79:201-210.

43 Cahn W, Hulshoff Pol HE, Bongers M, Schnack HG, Mandl RC, Van Haren NE, Durston S: Brain morphology in antipsychotic-naive schizophrenia: a study of multiple brain structures. Br J Psychiatry Suppl 2002;43:s66-s72.
44 Gur RE, Cowell P, Turetsky BI, Gallacher F, Cannon T, Bilker W, Gur RC: A follow-up magnetic resonance imaging study of schizophrenia: relationship of neuroanatomical changes to clinical and neurobehavioral measures. Arch Gen Psychiatry 1998;55: 145-152.

45 Salokangas RK, Cannon T, Van Erp T, Ilonen T, Taiminen T, Karlsson H, Lauerma H, Leinonen KM, Wallenius E, Kaljonen A, Syvalahti E, Vilkman H, Alanen A, Hietala J: Structural magnetic resonance imaging in patients with first-episode schizophrenia, psychotic and severe non-psychotic depression and healthy controls: results of the schizophrenia and affective psychoses (SAP) project. Br J Psychiatry Suppl 2002;43:s58s65.

46 Melle I, Friis S, Hauff E, Vaglum P: Social functioning of patients with schizophrenia in high-income welfare societies. Psychiatr Serv 2000;51:223-228.

47 Wieselgren IM, Lindstrom LH: A prospective 1-5 year outcome study in first-admitted and readmitted schizophrenic patients; relationship to heredity, premorbid adjustment, duration of disease and education level at index admission and neuroleptic treatment. Acta Psychiatr Scand 1996;93:9-19.

48 Staal WG, Hulshoff Pol HE, Kahn RS: Outcome of schizophrenia in relation to brain abnormalities. Schizophr Bull 1999;25:337348 .

49 Mitelman SA, Newmark RE, Torosjan Y, Chu KW, Brickman AM, Haznedar MM, Hazlett EA, Tang CY, Shihabuddin L, Buchsbaum MS: White matter fractional anisotropy and outcome in schizophrenia. Schizophr Res 2006;87:138-159.

50 Highley JR, Esiri MM, McDonald B, Roberts HC, Walker MA, Crow TJ: The size and fiber composition of the anterior commissure with respect to gender and schizophrenia. Biol Psychiatry 1999;45:1120-1127.

51 Highley JR, Esiri MM, McDonald B, Cortina-Borja $\mathrm{M}$, Herron BM, Crow TJ: The size and fibre composition of the corpus callosum with respect to gender and schizophrenia: a post-mortem study. Brain 1999;122: 99-110.

52 Delisi LE, Bertisch HC, Szulc KU, Majcher M, Brown K, Bappal A, Ardekani BA: A preliminary DTI study showing no brain structural change associated with adolescent cannabis use. Harm Reduct J 2006;3:17.

53 Ashburner J, Friston KJ: Why voxel-based morphometry should be used. Neuroimage 2001;14:1238-1243. 